\title{
Origin of fibronectin in epiretinal membranes of proliferative vitreoretinopathy and proliferative diabetic retinopathy
}

\author{
Salvatore Grisanti, Klaus Heimann, Peter Wiedemann
}

\begin{abstract}
Fibronectins, high molecular multifunctional glycoproteins of the extracellular matrix and plasma, have been a popular area of research in the pathogenesis of proliferative disorders of the retina. Several immunohistochemical studies have revealed that fibronectin is a major constituent of epiretinal membranes and that the cell types involved in proliferative intraocular disorders may synthesise it. However, owing to the fact that plasma and cellular fibronectin are similar in their overall structure, the origin of fibronectin in epiretinal membranes has not yet been clearly defined. In this study, we used two monoclonal antibodies: FN-3, which recognises an extra domain present in the cellular but not plasma form of fibronectin; and FN-4, which reacts with an antigenic site on both plasma and cellular fibronectin. In 37 epiretinal membranes obtained from eyes with proliferative vitreoretinopathy and proliferative diabetic retinopathy, we demonstrated the presence of cellular fibronectin, thus indicating local production. The significantly stronger and positive immunostain with $\mathrm{FN}-4$ in the same specimens suggests the colocalisation of plasma fibronectin, that may be derived from the breakdown of the blood-retinal barrier and trapped in membranes during their formation. In pathological vitreous we demonstrated both types of fibronectin by western blot analysis. (BrF Ophthalmol 1993; 77: 238-242)
\end{abstract}

The uncontrolled growth and proliferation of several types of non-neoplastic cells at the vitreoretinal interface is the common final pathway of the eye's reaction towards vitreoretinal alteration. In proliferative vitreoretinopathy (PVR) the development of tractional membranes leading to a significant and definitive visual loss seems to be caused by a complex series of cellular and biochemical interactions similar to the proliferative fibroblastic phase of physiological wound healing. Fractional epiretinal membranes also develop during the course of proliferative diabetic retinopathy (PDR).

Fibronectins constitute a class of high molecular weight proteins $(440 \mathrm{kD})$ which are widely present on the cell surface of several types of cells, in basement membranes, in stromal matrices, and in plasma. ${ }^{1}$ The multiple biological functions of these glycoproteins result from their ability to promote cell migration adhesion and differentiation, to enhance the synthesis of cytoskeletal proteins and stabilisation of the extracellular matrix, and finally to participate in the process of phagocytosis, wound healing, and blood coagulation. ${ }^{23}$ It is also well known that fibronectins have a domain with special affinity to collagen, heparin, fibrin, fibronogen, hyaluronic acid, and complement factor Clq. These interactions may be of particular importance in the development of contractile membranes at the vitreoretinal interface in PVR and PDR as they allow the cells to attach to the matrix.

Research has focused on the involvement of fibronectins in proliferative intraocular disorders as they were found to be chemotactic for the retinal pigment epithelial (RPE) cells, ${ }^{4}$ present in vitreous aspirates from patients with PVR and PDR obtained during vitrectomy, ${ }^{56}$ and identified as the major constituent of young epiretinal membranes. ${ }^{789}$ However, neither these findings, which introduced fibronectin as an important pathogenetic factor into the disease concept, nor the fact that the major cell types involved in PVR and PDR may synthesise fibronectin, ${ }^{31011}$ could clearly define the origin of fibronectin in epiretinal membranes.

Recently, the first approach of recognition of a local production of fibronectin in epiretinal membranes was made by Immonen $e t$ al, ${ }^{12}$ who demonstrated the occurrence of cellular fibronectin in a small number of epiretinal membranes, and by Hiscott et $a l,{ }^{13}$ who showed fibronectin related mRNA by in situ hybridisation. In this study we directly identified fibronectin located in contractile membranes using two monoclonal antibodies (FN-3 and FN-4) that recognise two different antigenic sites on the cell-associated fibronectin; one of them (the FN-3 determinant) is not found in human plasma fibronectin. ${ }^{14}$

\section{Materials and methods}

SAMPLES AND CELL CULTURES

Vitreoretinal traction membranes were obtained from patients undergoing surgical treatment for

Table 1 Expression of cellular fibronectin $(F N-3)$ and cellular/plasma fibronectin (FN-4) in surgically excised human epiretinal membranes (The staining intensity for fibronectins was graded as strong $(++)$, weak $(+)$, absent (-))

\begin{tabular}{lrll}
\hline Diagnosis & No & $\begin{array}{ll}F N-3 \\
-/+/++\end{array}$ & $\begin{array}{l}F N-4 \\
-/+1++\end{array}$ \\
\hline Idiopathic PVR & 7 & $2 / 5 / 0$ & $0 / 3 / 4$ \\
Traumatic PVR & 18 & $8 / 9 / 1$ & $0 / 11 / 7$ \\
Macular pucker & 7 & $3 / 4 / 0$ & $0 / 5 / 2$ \\
PDR & 5 & $2 / 2 / 1$ & $0 / 3 / 2$ \\
\hline
\end{tabular}

$\mathrm{PVR}=$ proliferative vitreoretinopathy $\mathrm{PDR}=$ proliferative diabetic retinopathy

Cologne, Germany.

Accepted for publication

9 December 1992 
traction retinal detachment due to PVR and PDR and processed as described elsewhere. ${ }^{9} \mathrm{We}$ included 18 membranes from eyes with traumatic PVR, seven with idiopathic PVR, seven with macular pucker, and five specimens from eyes with PDR. Central vitreous from donor eyes for keratoplasty was aspirated within 6 hours after death and immediately stored at $-20^{\circ} \mathrm{C}$. Vitreous aspirates were obtained from patients with idiopathic $(n=3)$ and traumatic PVR $(n=3)$, and PDR $(n=3)$ by needle aspiration from the centre of the vitreal cavity before vitrectomy. Pooled human serum (Sigma S-2257) and purified fibronectin from human plasma (Sigma F-2006) were also examined.

\section{BUFFERS AND REAGENTS}

The following buffers and solutions were employed: phosphate buffered saline (PBS) (pH 7·3). PBS-Tween containing $0 \cdot 05 \%$ (blot staining) Tween 20 (Serva 37470); fast red TR (Sigma F-2768), naphthol AS-MX phosphate (Sigma N-4875) stain buffer ( $\mathrm{pH} \mathrm{8 \cdot 2).}$

\section{IMMUNOHISTOCHEMICAL ANALYSIS OF} EPIRETINAL MEMBRANES

The preparation of slides for immunohistochemistry and the general immunostaining

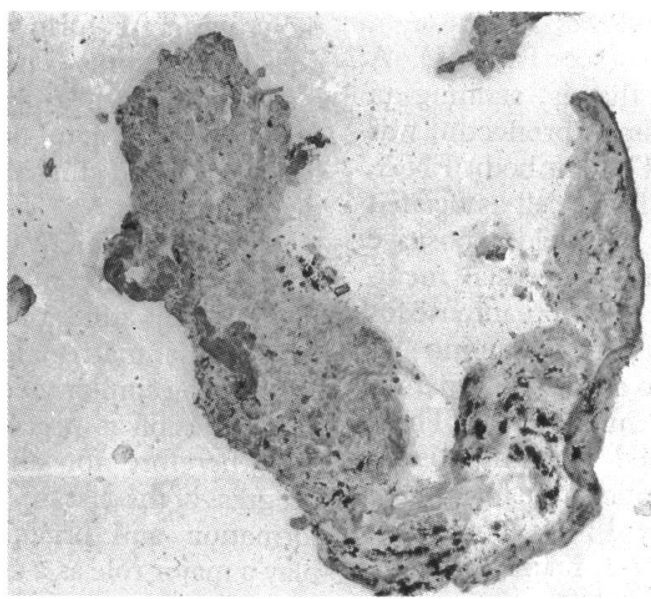

Fig $1 A$

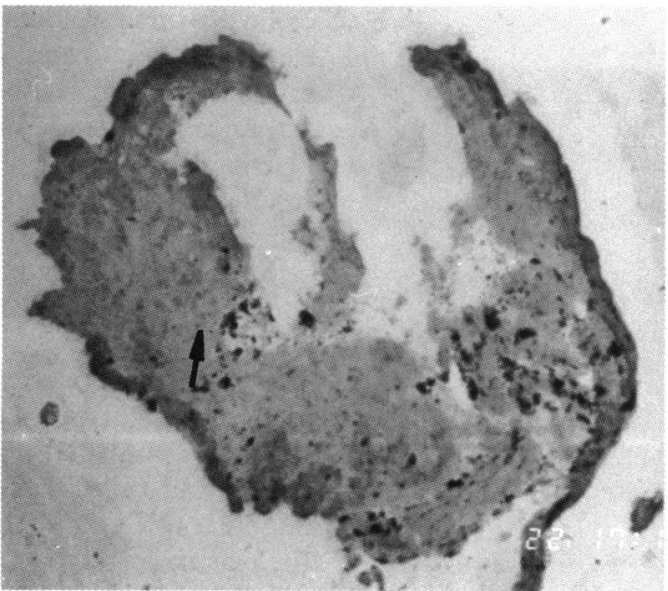

Fig $1 B$

Figure 1 Alkaline phosphatase staining of serial frozen sections of surgically excised human epiretinal membranes with $F N-4$ $(A, C)$, and $F N-3(B, D)$. Phosphatase converts fast red substrate to a red product. Counterstaining with haematoxylin and eosin shows cell nuclei (some marked by arrows). Magnification $A / B: \times 70 ; C / D \times 280$. technique of epiretinal membranes were performed as previously described..$^{95}$ For assay, all antibodies were diluted in PBS containing $0.5 \%$ bovine serum albumin. The following primary antibodies were used with the indicated dilutions: anti-human (cellular) fibronectin FN3, 1:10 (Cymbus Bioscience CBL 181); antihuman (cellular and plasma) fibronectin FN-4, 1:10 (Cymbus Bioscience CBL 182); anti-human fibronectin, 1:100 (Calbiochem 341643).

Primary antibody was labelled with a biotinstreptavidin system consisting of antimouse immunoglobin (1:500 Dakopatts E 418 01), and streptavidin conjugated with alkaline phosphatase (1:1000, Dakopatts D 39621 ).

This immunohistochemical staining was analysed and documented with a photomicrographic camera (MC 100, Zeiss, Germany).

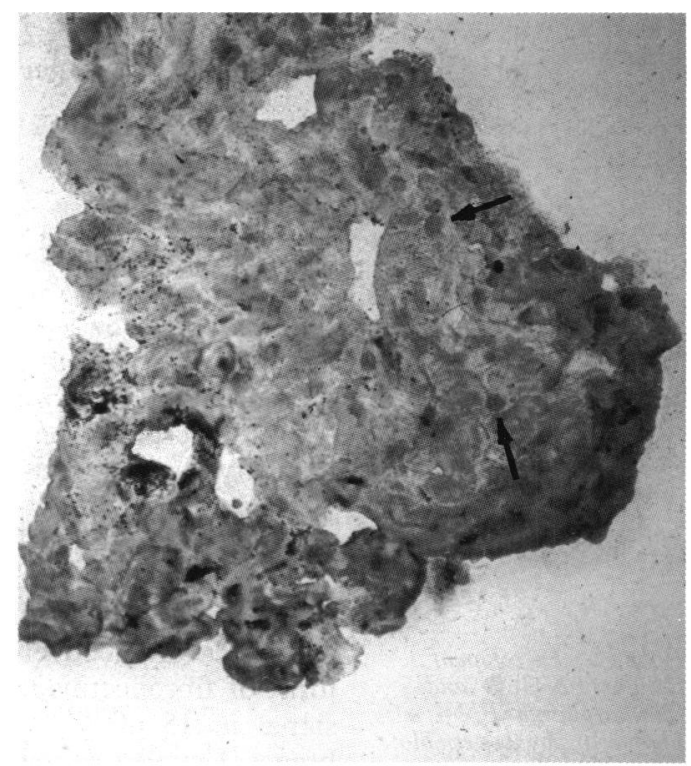

Fig 1C

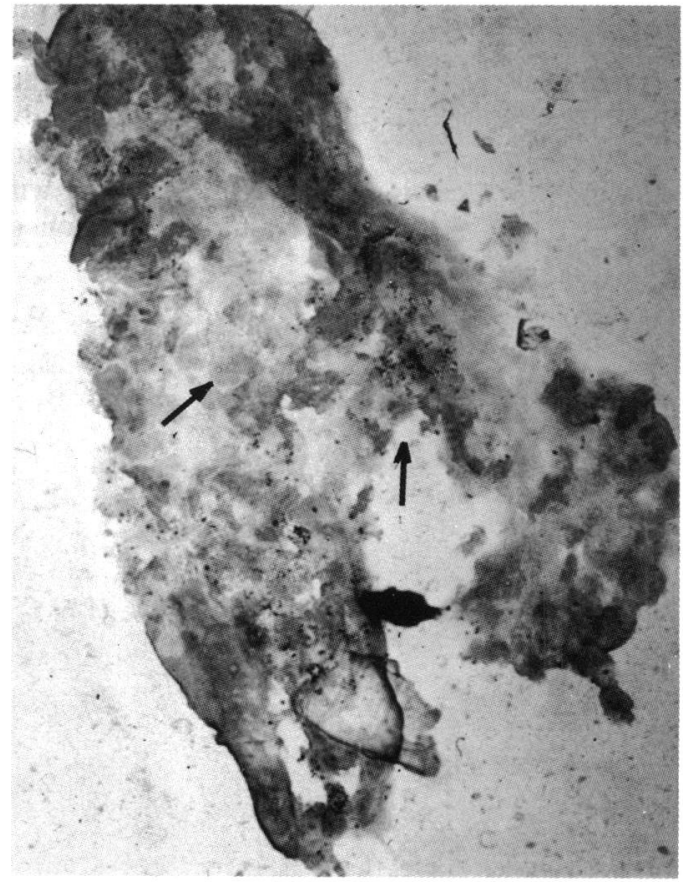

Fig $1 D$ 
Qualitative differences were concluded from serial sections.

\section{SDS-PAGE AND WESTERN BLOTTING}

The specificity of the primary antibodies against fibronectin was confirmed by sodium dodecylsulphate-polyacrylamide gel electrophoresis (SDS-PAGE) $)^{16}$ of human plasma fibronectin and subsequent western blot analysis. ${ }^{17}$ These techniques were also used for the examination of vitreous samples.

\section{CONTROLS}

As a means of negative control, parallel slides were stained with the first antibody substituted by non-immune IgG from mouse (Sigma I-5381) or rabbit (Sigma I-5006), both in 50\% higher concentrations. Positive controls of plasma fibronectin (Sigma S-2257) were achieved with SDS-PAGE and western blotting.

\section{Results}

\section{IMMUNOHISTOCHEMICAL MICROSCOPY OF} EPIRETINAL MEMBRANES

We examined 37 epiretinal membranes from patients with PVR $(n=32)$ or $\operatorname{PDR}(n=5)$ for the presence of two different antigenic determinants of fibronectins. The fibronectins stain label intensity was graded as follows: absent ( - , weak $(+)$, or strong $(++)$ (see Table 1). A positive signal for $\mathrm{FN}-4$ - that is, staining an epitope on cellular and plasma fibronectin, was seen in all 37 specimens. The antibody FN-3, which reacts selectively with the cell-associated form of fibronectins, ${ }^{14}$ did not yield a positive signal in 15 of 37 examined epiretinal membranes. Usually the staining was more intense for FN-4 than for FN-3. Negative staining for cellular fibronectin, but also a weaker signal for FN-4, was found in older membranes. These membranes are characterised by a diminished number of cells and fibrotic transformation. Staining with the antibody FN-4 showed, in contrast to staining with FN-3, mainly a homogeneous distribution of the glycoprotein. One from pathological vitreous. id (idiopathic) PVR; tr (traumatic) PVR; PDR (proliferative diabetic retinopathy).

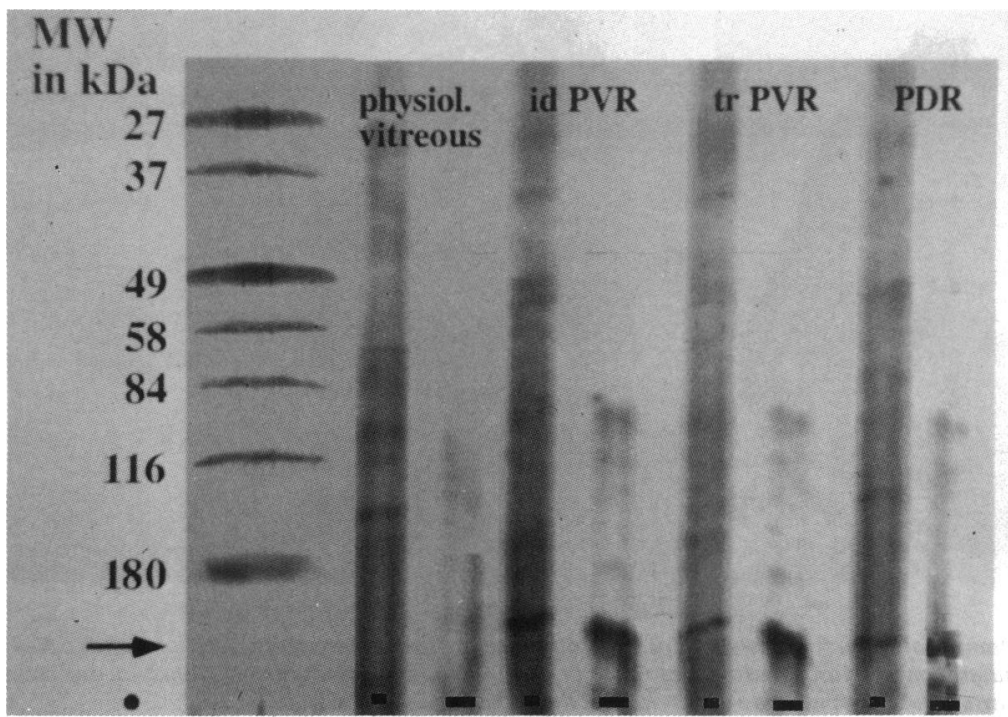

can see areas which stained with $\mathrm{FN}-4$, but did not stain with FN-3 (Fig 1).

WESTERN BLOT

Antibody FN-4 clearly precipitated fibronectin in pooled human plasma and purified fibronectin while antibody FN-3 did not. ${ }^{14}$ No fibronectins could be detected by western blot in normal human postmortem vitreous (Fig 2).

We analysed vitreous samples from patients with idiopathic $(n=3)$ and traumatic PVR $(n=3)$ and PDR $(n=3)$. Although cellular or cellassociated fibronectin, respectively, forms higher oligomers than plasma fibronectin and is therefore much less soluble, ${ }^{18} 19$ its presence could be demonstrated in the vitreous of the pathological eyes by the selective $\mathrm{FN}-3$ reactivity and different stain pattern with regard to $\mathrm{FN}-4$ reactivity (Fig 2). While cellular fibronectin can be recognised as a narrow band positioned in a higher molecular weight area than the $180 \mathrm{kD}$ marker protein, FN-4 (cellular and plasma) staining revealed a broader band on the same molecular weight position. One vitreous sample in the traumatic PVR and one in the PDR group did not contain enough cellular fibronectin to be visualised by the western blotting technique.

\section{Discussion}

The pathogenesis of PVR is characterised by a dispersion of cells into the vitreal cavity, where they proliferate, form conglomerates, and finally detach the retina. The disease progression has many features of a maladapted wound healing process, and there is extensive experimental histological and clinical evidence which supports this hypothesis. ${ }^{20}$

Although different interactions are important in initiating and controlling PVR and PDR, the phase of cell adhesion and interaction with the extracellular matrix and matrix proteins seems to be based on similar mechanisms in both diseases. Cell adhesion is preconditional for the growth and therefore for all following steps in the progress of the disease. In this stage of membrane formation and organisation, fibronectin may play a major role as a chemoattractant ${ }^{4}$ as well as an adhesion-promoting factor providing a temporary scaffold for cell migration.

Two principal forms of fibronectins that are similar in their overall structure but not identical in their chemical composition ${ }^{21}$ and biological activities ${ }^{22} 23$ were described. One has identified plasma fibronectin produced by liver cells $\mathrm{s}^{24}$ and cellular fibronectin produced by fibroblasts and other cell types. ${ }^{18}$ The differences between cellular and plasma fibronectins, which are encoded by a single gene, ${ }^{2125}$ seem to arise from post-transcriptional events via alternative splicing of fibronectin mRNA. ${ }^{26-28}$ Fibronectins form dimers of two subunit polypeptides (200$250 \mathrm{kD}$ ) connected near one end by disulphide bonds, but cellular fibronectin can form higher oligomers $^{1829}$ and is therefore less soluble than the plasma form.

Several studies have demonstrated that the major cell types involved in PVR and PDR - for example, RPE cells and fibroblasts, may synthesise fibronectin. ${ }^{3111}$ Since it has been 
demonstrated that plasma fibronectin can be deposited in tissues and incorporated into the cellular matrix, ${ }^{30}$ it has not been possible to identify the definitive source of fibronectin in tissue sections ${ }^{31}$ and therefore in epiretinal membranes, by means of polyclonal antisera.

Recently, the first approach of recognition of a local production in fibronectin in epiretinal membranes was made by Hiscott et al, ${ }^{13}$ who showed variable labelling of retinal pigment epithelium, retinal glia, and fibroblast-like cells in membranes at the vitreoretinal junction with a fibronectin mRNA probe. This observation correlates with the fact that RPE cells in vitro produce and deposit fibronectin in the extracellular matrix, ${ }^{10}$ and supports our findings of a direct identification of cellular fibronectin in epiretinal membranes that was also confirmed by Immonen et al. ${ }^{12}$

In situ hybridisation can show 'up-regulation' or activation of fibronectin production, respectively, and therefore demonstrates a productive disease stage. However, it will fail during 'down-regulation' or stagnation of production respectively, although a previous glycoprotein production may have induced a participation of locally synthesised cellular fibronectin in membrane formation. Owing to the fact that the direct identification of cellular, locally produced fibronectin can fill this information gap, the combination with the fibronectin mRNA probe would optimise the observation of stage and progress of the disease.

The significantly stronger positive immunostain with FN-4 and the occasionally negative immunostain with FN-3 and positivity with FN4 in the same specimens suggests the colocalisation of plasma fibronectins, that enters the eye after breakdown of the blood-retinal barrier and is trapped in membranes during their formation. The great difference between FN-4 and FN-3 staining in all membranes (for example, as in Fig 1) suggests that fibronectin in epiretinal membranes is predominantly plasma derived. Additionally, we observed the presence of both plasma and cellular fibronectin mostly in younger (only a few months), cell-rich membranes than in older fibrotic membranes.

Although several studies suggested that the particularly high vitreous fibronectin levels seen in patients with proliferative vitreoretinal disease are derived from plasma fibronectin following breakdown of the blood-retinal barrier, ${ }^{4-6}$ a contributing local production could not be excluded. Despite the small number of the analysed vitreal specimens in our study, we demonstrated the presence of cellular fibronectin by western blot. Owing to the fact that the western blot technique is qualitative rather than quantitative, we cannot assess the level of contribution by intravitreal production. However, lower levels of cellular fibronectin than of plasma fibronectin in epiretinal membranes, and the fact that cellular fibronectin is much less soluble than the plasma form, suggest a smaller contribution of locally produced glycoprotein.

The pathophysiological role of plasma fibronectin may be based on its quality as a structural protein. Circulating plasma fibronectin may serve as a building block for the early assembly of extracellular matrix by providing the possibility of attachment to vitreous collagen. In comparison with it cellular fibronectin seems to play its pivotal part in the regulation of cellular events ${ }^{22}$ enhancing the autonomity in membrane growth and transformation.

This study was supported by the Retinovit Foundation and the Deutsche Forschungsgemeinschaft (Wi880/3-2), and was presented in part at the ARVO meeting.

The authors would like to thank Mrs B Martiny for skilful technical assistance.

1 Yamada K, Olden K. Fibronectins - adhesive glycoproteins of cell surface and blood. Nature 1978; 275: 179-84.

2 Yamada K, Akiyama S, Hasegawa T, Hasegawa E, Humphries $M$, Kennedy $\mathrm{D}$, et al. Recent advantages in research on fibronectin and other cell attachment proteins. f Cell Biochem 1985; 28: 79-97.

3 Akiyama S, Yamada K. Fibronectin. Adv Enzymol Relat Areas Mol Biol 1987; 59: 1-57.

4 Campochiaro P, Jerdan J, Glaser B. Serum contains chemoattractants for human retinal pigment epithelial cells. Arch Ophthalmol 1984; 102: 1830-3.

5 Campochiaro P, Jerdan J, Glaser B, Cardin A, Michels R. Vitreous aspirates from patients with proliferative vitreoVitreous aspirates from patients with proliferative vitreo-
retinopathy stimulate retinal pigment epithelial cell migration. Arch Ophthalmol 1985; 103: 1403-5.

6 Weller M, Wiedemann P, Heimann K, Zilles K. The significance of fibronectin in vitreoretinal pathology. A critical evaluation. Graefes Arch Clin Exp Ophthalmol 1988; 226: 294-8.

7 Hiscott P, Grierson I, McLeod D. Natural history of fibrocellular epiretinal membranes. A quantitative, autoradiographic, and immunohistochemical study. $\mathrm{Br} \mathcal{F} O$ phthalmo 1985; 69: 810-23.

8 Scheiffarth O, Kampik A, Gunther $H$, von-der-Mark $K$. Proteins of the extracellular matrix in vitreoretinal membranes. Graefes Arch Clin Exp Ophthalmol 1988; 226: $357-61$

9 Weller $M$, Heimann $K$, Wiedemann P. Immunochemical studies of epiretinal membranes using APAAP complexes: evidenee for macrophage involvement in traumatic proliferative vitreoretinopathy. Int Ophthalmol 1988; 11: 181-6.

10 Campochiaro $P$, Jerdan J, Glaser B. The extracellular matrix of human retinal pigment epithelial cells in vivo and its 21 .

11 Alitalo K, Tapani H, Vaheri A. Fibronectin is produced by human macrophages. $\mathcal{F}$ Exp Med 1980; 151: 602-13.

12 Immonen I, Tervo K, Virtanen I, Laatikainen L, Tervo T. Immunohistochemical demonstration of cellular fibronectin and tenascin in human epiretinal membranes. Acta Ophthaland tenascin in human

13 Hiscott P, Waller H, Grierson I, Butler M, Scott D. Local production of fibronectin by ectopic human retinal cells. Cell Tissue Res 1992; 267: 185-92.

14 Keen J, Chang SE, Taylor-Papadimitriou J. Monoclonal antibodies that distinguish between human cellular and plasma fibronectin. Mol Biol Med 1984; 2: 15-27.

15 Cordell J, Falini B, Erber W, Ghosh A, Abdulaziz Z, MacDonald S, et al. Immunoenzymatic labelling of monoclonal antibodies using immune complexes of alkaline phosphatase and monoclonal antialkaline phosphatase (APAAP complexes). F Histochem Cytochem 1984; 32: 219 29.

16 Görg A, Postel W, Westermaier R, Righetti P. Gel gradient electrophoresis, isoelectric focussing and two-dimensional techniques in horizontal, ultrathin polyacrylamide layers. techniques in horizontal, ultrathin polyac

17 Towbin H, Gordon J. Immunoblotting and dot immunobinding - current status and outlook. F Immunol Methods 1984; 72 : $313-40$ 18 Hynes R, Destree A. Extensive disulfide bonding at the
mammalian cell surface. Proc Natl Acad Sci USA 1977; 74: 2855-9.

19 dArdenne A, McGee J. Fibronectin in disease. F Pathol 1984; 142: 235-51.

20 Weller M, Heimann K, Wiedemann P. Proliferative vitreoretinopathy - is it anything more than wound healing at the retinopathy - is it anything more than wound heal
wrong place? Int Ophthalmol 1990; 14: 105-17.

21 Hayashi M, Yamada K. Differences in domain structure between plasma and cellular fibronectins. Biol Chem 1981; 256: 11292-300.

22 Yamada K, Kennedy D. Fibroblast cellular and plasma fibronectins are similar but not identical. $\mathcal{f}$ Cell Biol 1979; 80: 492-8.

23 Vartio $T$, Laitinen $L$, Närvänen $O$, Cutolo $M$, Thornell LE, Zardi $\mathrm{L}$, et at. Differential expression of the ED sequencecontaining form of cellular fibronectin in embryonic and adult human tissues. $\mathcal{F}$ Cell Sci 1987; 88: 419-30.

24 Tamkun J, Hynes R. Plasma fibronectin is synthesized and secreted by hepatocytes. F Biol Chem 1983; 258: 4641-7.

25 Atherton B, Hynes R. A difference between plasma and cellular fibronectin located by monoclonal antibodies. Cell 1981; 25: 133-41.

26 Kornblitt A, Vide-Pedersen K, Baralle F. Isolation and characterization of CDNA clones for human and bovine fibronectins. Proc Natl Acad Sci USA 1983; 80: 3218-22. 
molecular cloning evidence for two mRNA species differing by an integral segment coding for a structural domain

$E M$ Schwarzbauer J, Tamkun J, Lemischka I, Hynes R. Three
. different fibronectin mRNAs arise by the alternative splicing with the coding region. Cell 1983; 35: 421-31.

29 Engwald E, Ruoslauti E, Miller E. Affinity of fibronectin to collagen of different genetic types and to fibrinogen. $\mathcal{F}$ Exp Med 1978; 147: 1584-95.

30 Oh E, Pierschbacher M, Ruoslahti E. Deposition of plasma fibronectin in tissues. Proc Natl Acad Sci USA 1981; 78: 3218-21.

31 Stenman S, Vaheri A. Fibronectin in human solid tumors. Int f Cancer 1981; 27: 427-35. 